\title{
Prevalence of antimicrobial resistant bacteria from adult ICUs and the Burns unit at a large tertiary hospital in Durban
}

\author{
K. Swe Swe-Han, Y. Coovadia \\ Department of Medical Microbiology, Academic Complex, NHLS/ UKZN, Durban, KZN \\ doi: 10.3396/ijic.V6i2.015.10
}

\section{Introduction}

The prevalence of antimicrobial resistant bacteria vary between wards in Hospitals influenced by selective pressure of antimicrobials used in a hospital. Infection control practices within the hospital, type of hospital and risk factors in a patient. ${ }^{1}$ This information is particularly valuable in choosing empirical antimicrobial therapy for serious hospital acquired infections. ${ }^{2}$

\section{Aim}

This study was undertaken, to determine the prevalence of antimicrobial resistant bacterial isolates from adult medical and surgical ICUs, trauma ICU (TICU), Cardiothoracic ICU (CTC ICU) and the burns unit in a large tertiary hospital in Durban, KwaZulu Natal and to determine the prevalence of antimicrobial resistance in selected bacterial isolates.

\section{Methods}

We analysed, over a three-month period [January to March 2008], the antimicrobial resistance profile of selected bacterial isolates, from adult ICUs and the Burns unit, to certain marker antibiotics. The study was based on laboratory records. The data was collected manually by Pathologist from all completed laboratory worksheets. The data were recorded as targeted common bacteria isolates, type of specimens, wards, date of sent, patients' identification, and antimicrobial susceptibility results. Study population was defined as patients with suspected infections from specific targeted wards (ICUs, Cardiothoracic unit and Burns unit) during the study period. The representative samples of suspected septic patients were sent by clinicians.

Only one representative isolate from each specimen per patient, regardless of clinical significant isolates, was included in the analysis. The bacterial isolates included in the analyses were Klebsiella pneumoniae, Pseudomonas aeruginosa, Acinetobacter species, and Staphylococcus aureus. The selected marker antibiotics were aminoglycosides (gentamicin and amikacin), beta lactams (piperacillin-tazobactam), fluroquinolone (ciprofloxacin), carbapenems (meropenem) and cloxacillin [methicillin].

Multi-Drug-Resistance [MDR] in the Gram negative isolates was defined as resistance to three or more first line classes (beta lactams, aminoglycoside, fluroquinolone) of antibiotics or resistant to carbapenem, ESBL-producing organisms, for that particular isolate. ${ }^{3}$

\footnotetext{
Corresponding author

K Swe Swe-Han, Department of Medical Microbiology, Academic Complex, NHLS/ UKZN, Durban, KZN
} 
The bacterial isolates were identified using standard laboratory techniques [API] and antimicrobial susceptibility was performed using the Kirby-Bauer method as recommended by CLSI, USA. Extended beta lactamase production was detected using the double disc diffusion method. ${ }^{4}$ Simple data analysis was performed based on laboratory data base.

There was total number of specific multidrug resistant isolates in specific target ward for a study time period as numerator and total number of specific organisms from the specific ward for a study time period as denominator. Prevalence rate is a proportion and can be expressed as a percentage.

\section{Results and Discussion}

The prevalence (proportion) of MRSA in these units was $66 \%, 48 \%, 88 \%, 52 \%$ and $67 \%$ respectively (Table I \& figure 1). Similarly, we analysed for other targeted MDR organisms (ESBL+ K. pneumoniae, MDR Acinetobacter spp and MDR P. aeruginosa) (Table I).

Table I: Prevalence of antimicrobial resistant bacteria for the 3 months Period Jan- March 2008

\begin{tabular}{|c|c|c|c|c|c|}
\hline \multirow{3}{*}{$\begin{array}{l}\text { Wards } \\
\text { Burns }\end{array}$} & \multicolumn{5}{|c|}{ Bacterial isolates and resistance profile } \\
\hline & \multicolumn{2}{|c|}{ MRSA } & $\begin{array}{c}E S B L+ \\
\text { K. pneumoniae }\end{array}$ & $\begin{array}{c}M D R \\
\text { Acinetobacter spp }\end{array}$ & \multirow{2}{*}{$\begin{array}{c}\text { MDR } \\
\begin{array}{c}\text { Pseudomonas } \\
\text { aeruginosa }\end{array} \\
12 / 48 \quad(25 \%)\end{array}$} \\
\hline & $29 / 44$ & $(66 \%)$ & $2 / 5 \quad(40 \%)$ & $5 / 7 \quad(71 \%)$ & \\
\hline Medical \& surgical ICUA & $14 / 29$ & $(48 \%)$ & $24 / 29 \quad(83 \%)$ & $25 / 38 \quad(66 \%)$ & $3 / 23 \quad(13 \%)$ \\
\hline $\begin{array}{l}\text { Medical\& surgical ICUB, } \\
\text { neurosurgery }\end{array}$ & & $(88 \%)$ & $22 / 27 \quad(81 \%)$ & $\begin{array}{ll}6 / 9 & (67 \%)\end{array}$ & $4 / 29 \quad(14 \%)$ \\
\hline Trauma ICU & $15 / 29$ & $(52 \%)$ & $2 / 10 \quad(20 \%)$ & $13 / 22 \quad(59 \%)$ & $0 / 13 \quad(0 \%)$ \\
\hline Cardiothoracic unit & $2 / 3$ & $(67 \%)$ & $10 / 11 \quad(91 \%)$ & $1 / 2 \quad(50 \%)$ & $0 / 0 \quad(0 \%)$ \\
\hline
\end{tabular}

S. aureus (total no. of S. aureus isolates were 44, 29, 8, 29, 3 from Burns, ICU A, ICU B, T ICU and Cardiothoracic unit respectively as denominators)

MRSA (total no. of MRSA were 29, 14, 7, 15, 2 from Burns, ICU A, ICU B, T ICU and Cardiothoracic unit respectively as numerators) 
Although there were high percentage for some organisms, the total number of isolates (denominators) were $<10$. Because of these reasons, Figures 2, 4, 6 and 8 show number of isolates rather than percentages.

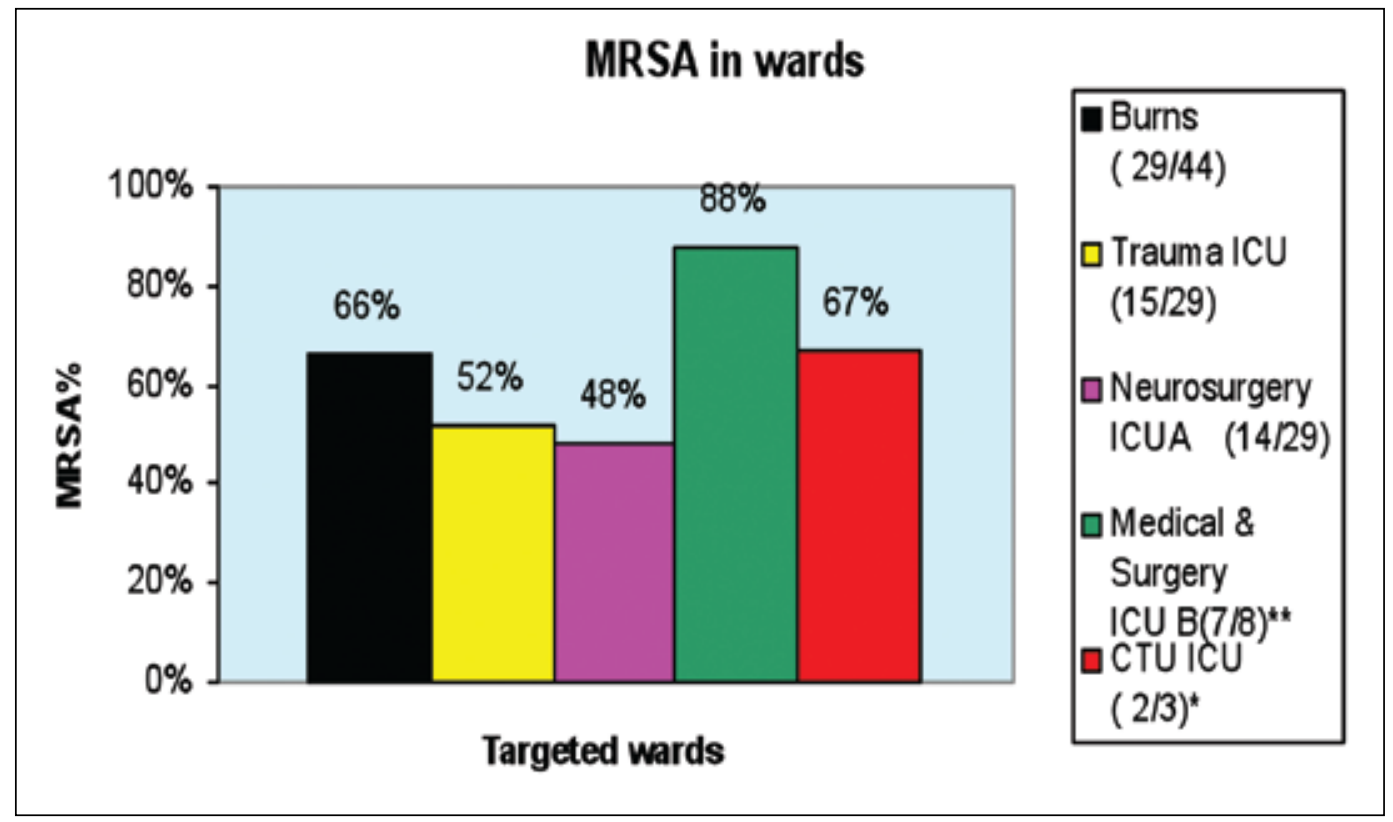

CTU ICU* - 2 MRSA out of $3 \mathrm{~S}$. aureus isolates from 3 different specimens of one patient. ICU $B^{* *}-7$ MRSA out of $8 \mathrm{~S}$. aureus isolates from different repeated specimens of 3 patients.

Figure 1. MRSA Histogram in 3 months study period (Jan- March 2008)

The number of MRSA varied from month to month in all selected wards (Figure 2). It was more problem in
Burns, T ICU, and ICU A for each month. In CTU ICU, 2 MRSA were isolated from one patient in February.

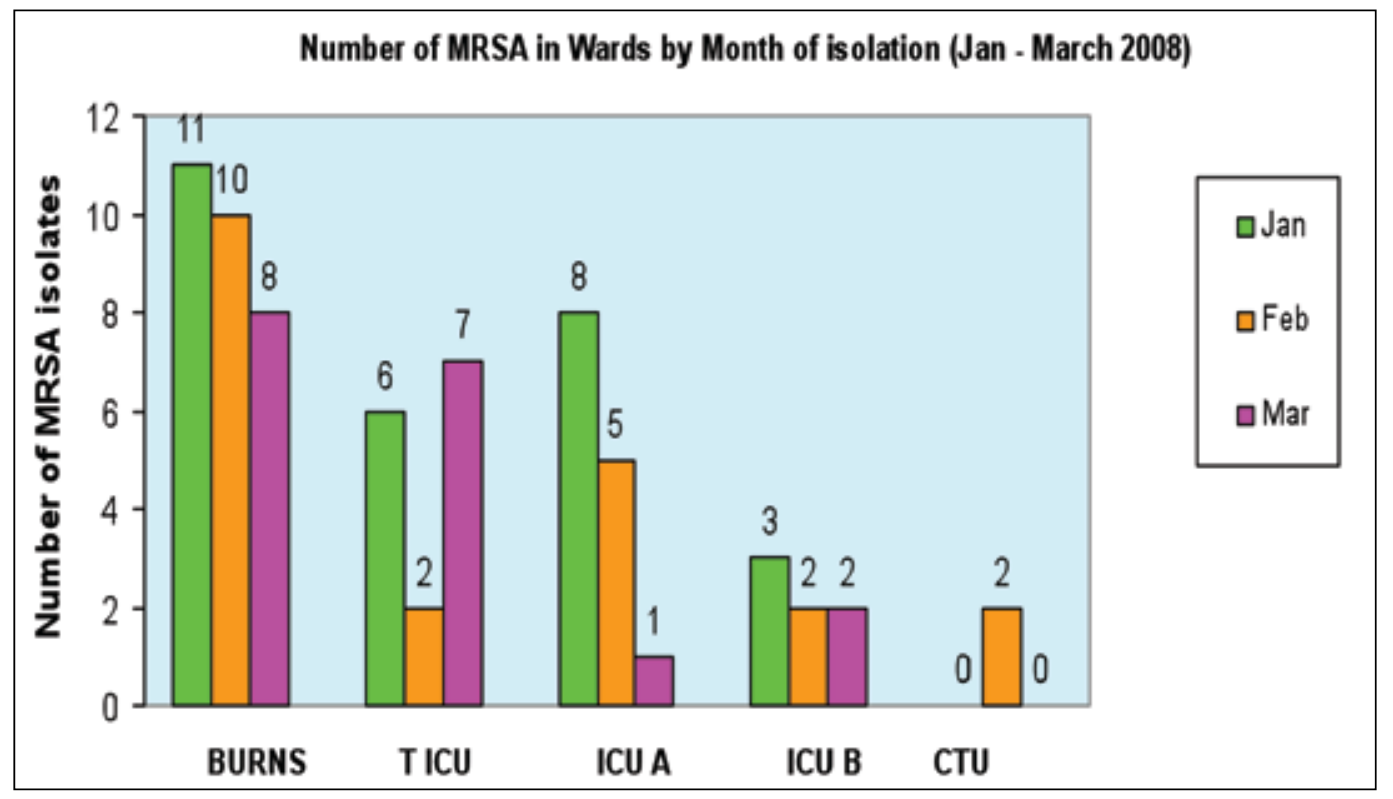

Figure 2. MRSA Histogram in different wards by month of isolation 
The prevalence /proportion of ESBL+ K.pneumoniae were high in ICU A, ICU B and CTU (Figure 3). The prevalence was higher in Burns than those in $\mathrm{T} \mathrm{ICU}$, but which was misleading for interpretation. It is statistically not acceptable if the denominator was $<10$.

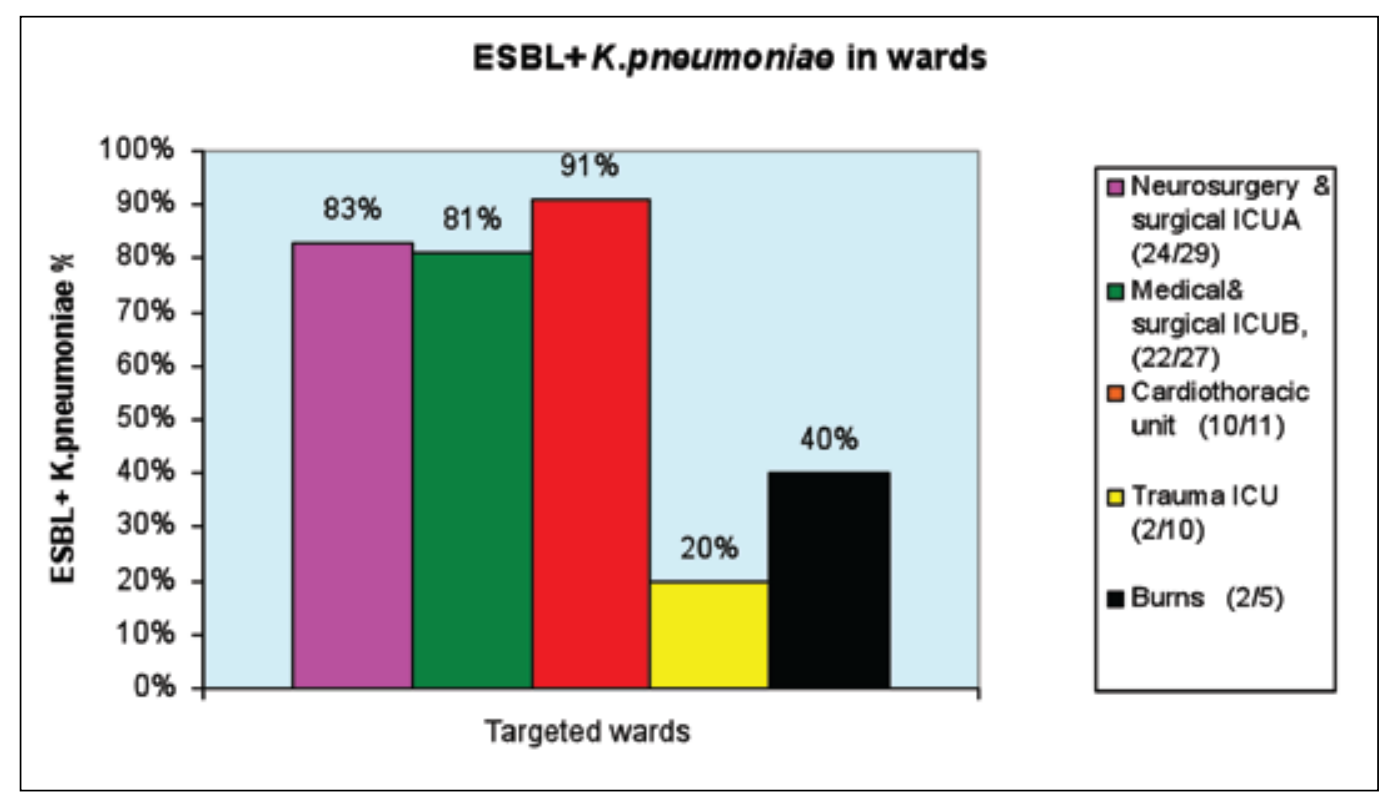

Figure 3. ESBL+ K. pneumoniae Histogram

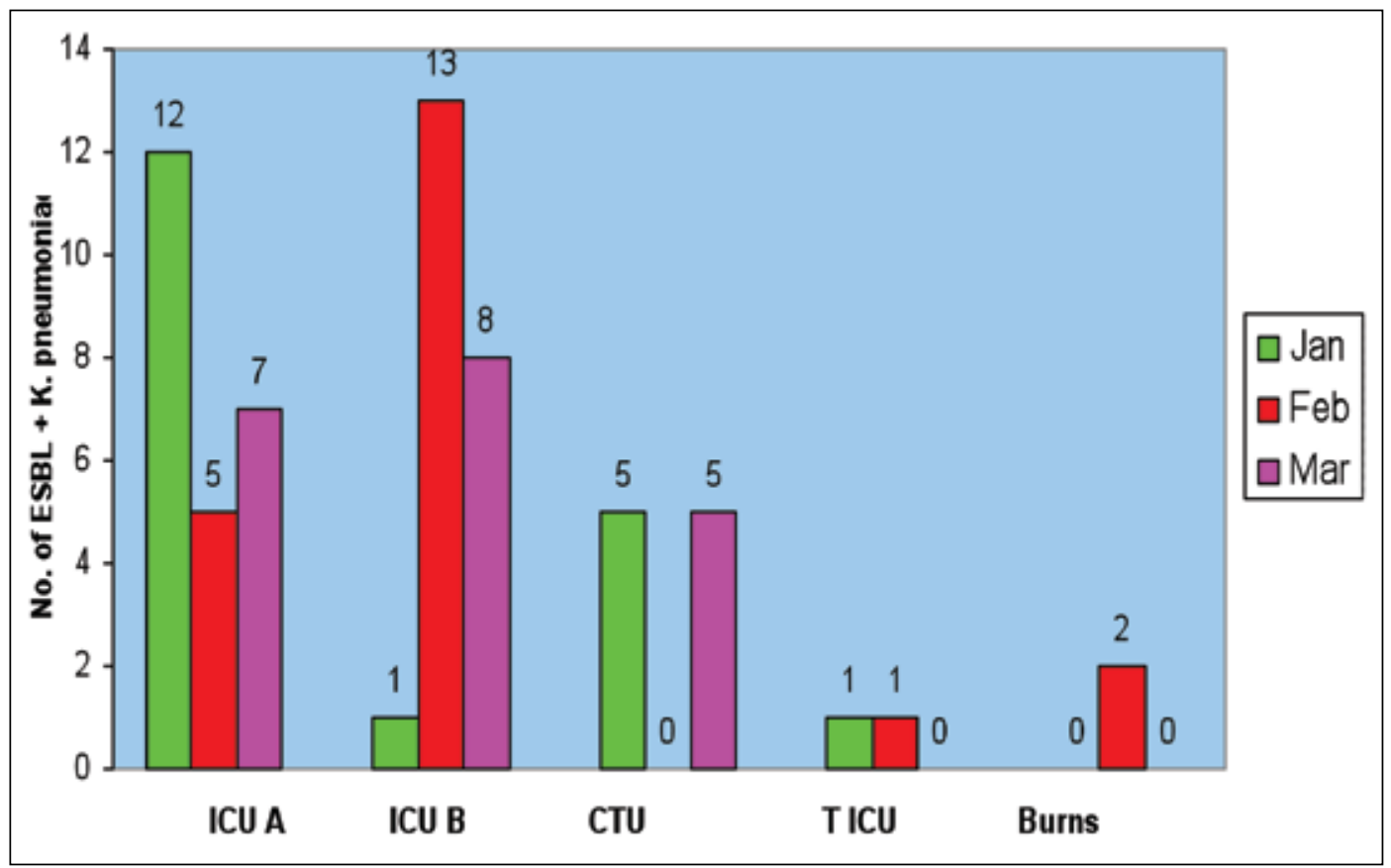

$2 E S B L+$ K. pneumoniae isolated from different specimen of one patient at Burns unit.

Figure 4. Number of ESBL+ K. pneumoniae in wards by month of isolation 
The prevalence / proportion of MDR Acinetobacter spp exceeded $50 \%$ (Figure 5 ) and it was more common in ICU A, T ICU regarding to number of denominator and numerator.
High number of MDR Acinetobacter spp isolate from all targeted wards in January (Figure 6).

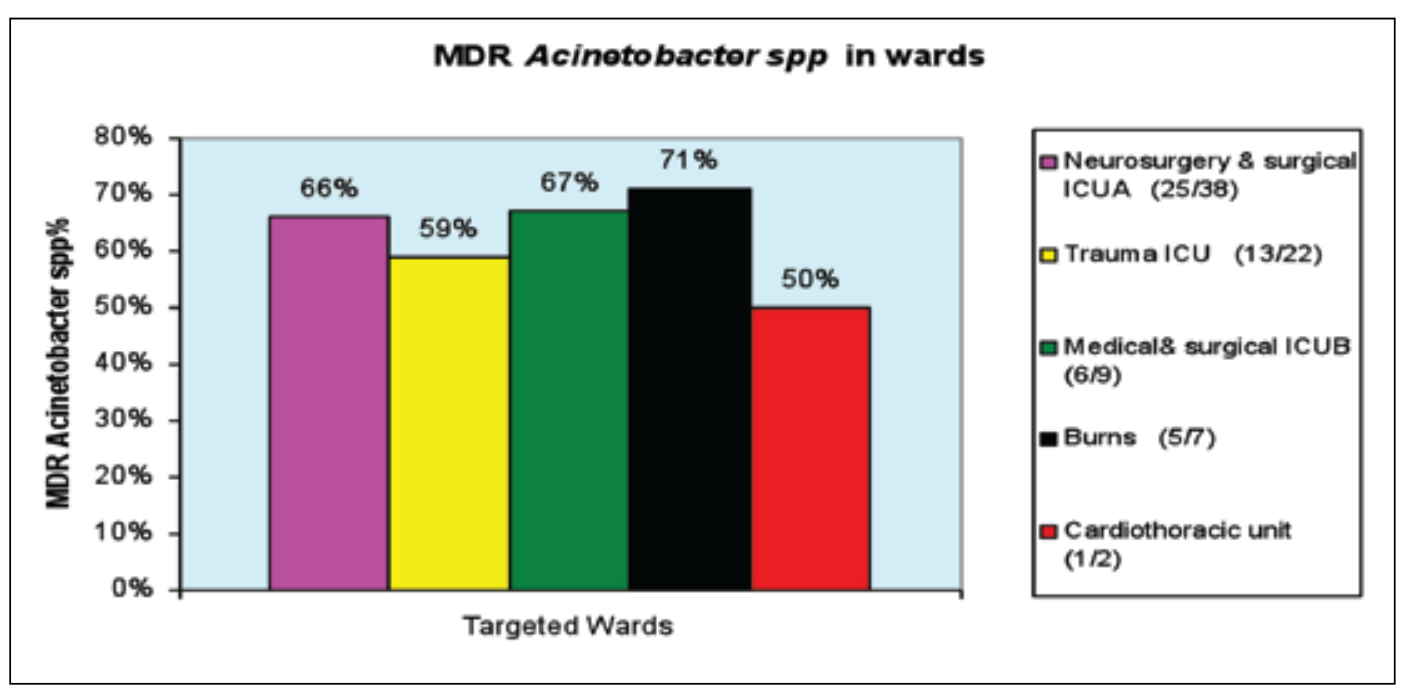

Figure 5. MDR Acinetobacter spp Histogram

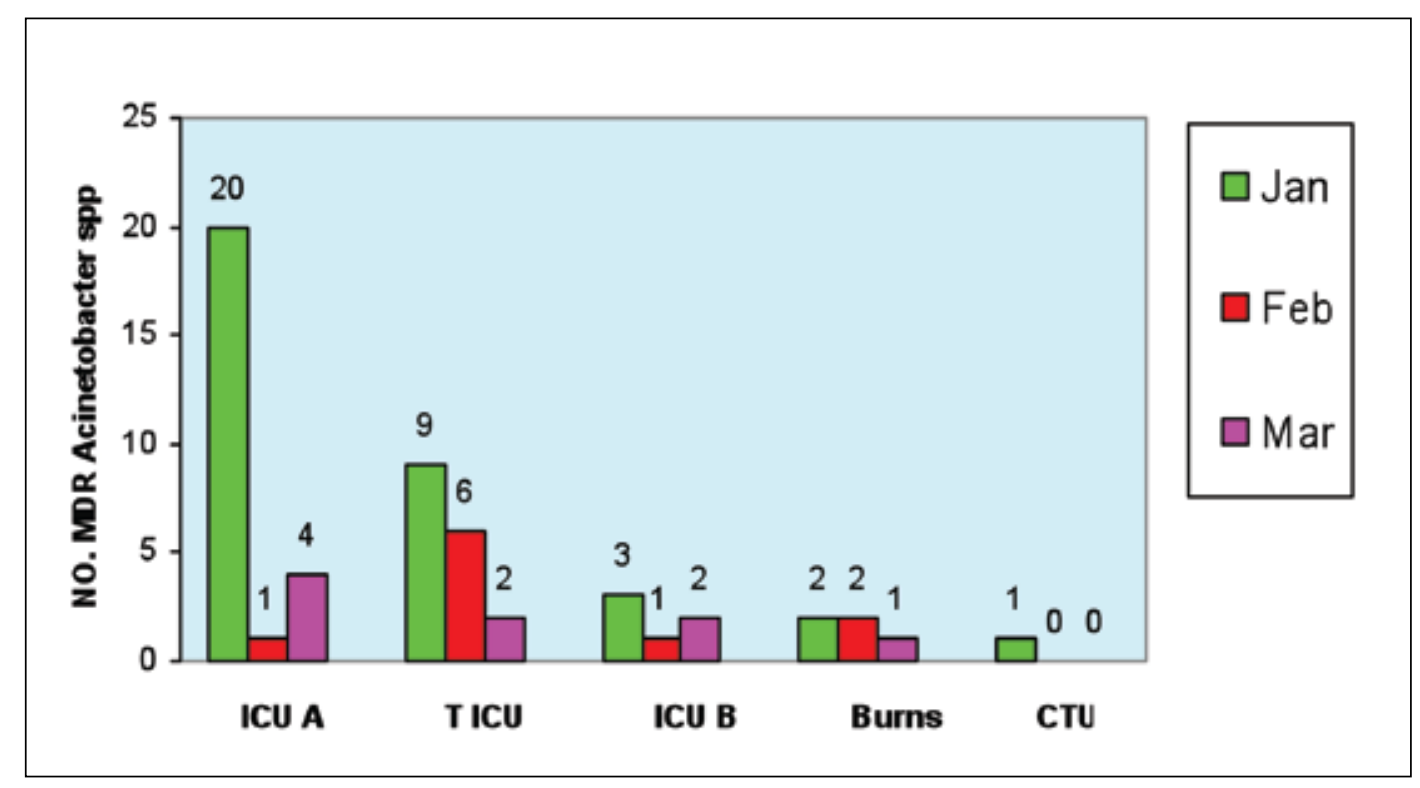

Figure 6. Number of MDR Acinetobacter spp in different wards by month of isolation 
The prevalence of MDR $P$. aeruginosa was highest in Burns and followed by ICU B and ICU A (Figure 7). There was $0 \%$ in both TICU and CTU ICU. The difference was no $P$. aeruginosa isolates at all in CTU within 3 months study periods. But sensitive $P$. aeruginosa isolated in TICU (See denominators).
MDR P. aeruginosa was consistently isolated in Burns unit within 3 months (Figure 8).

Antibiogram showed resistant \% of appropriate drugs was varied in each month in all targeted wards (Figure 9).

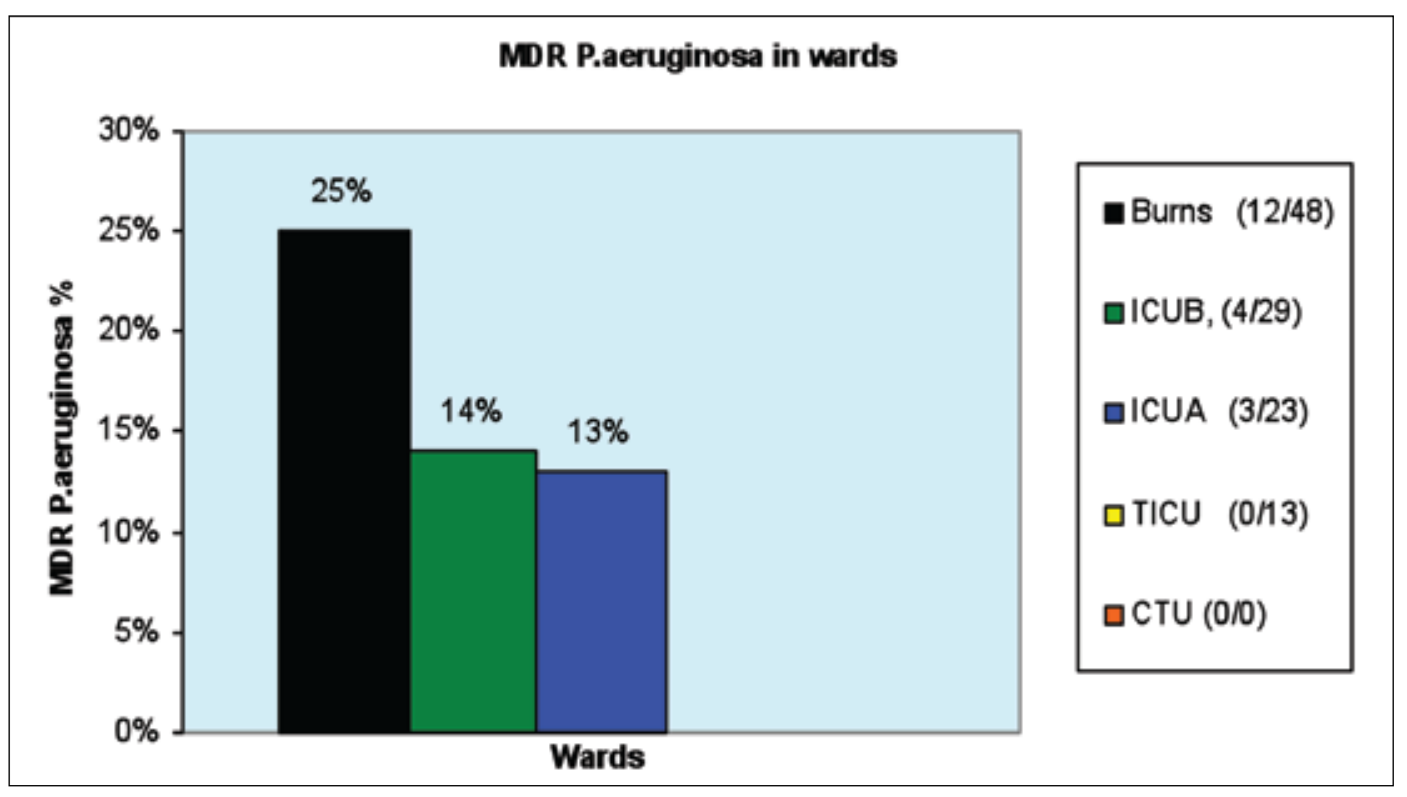

Figure 7. MDR Pseudomonas aeruginosa Histogram

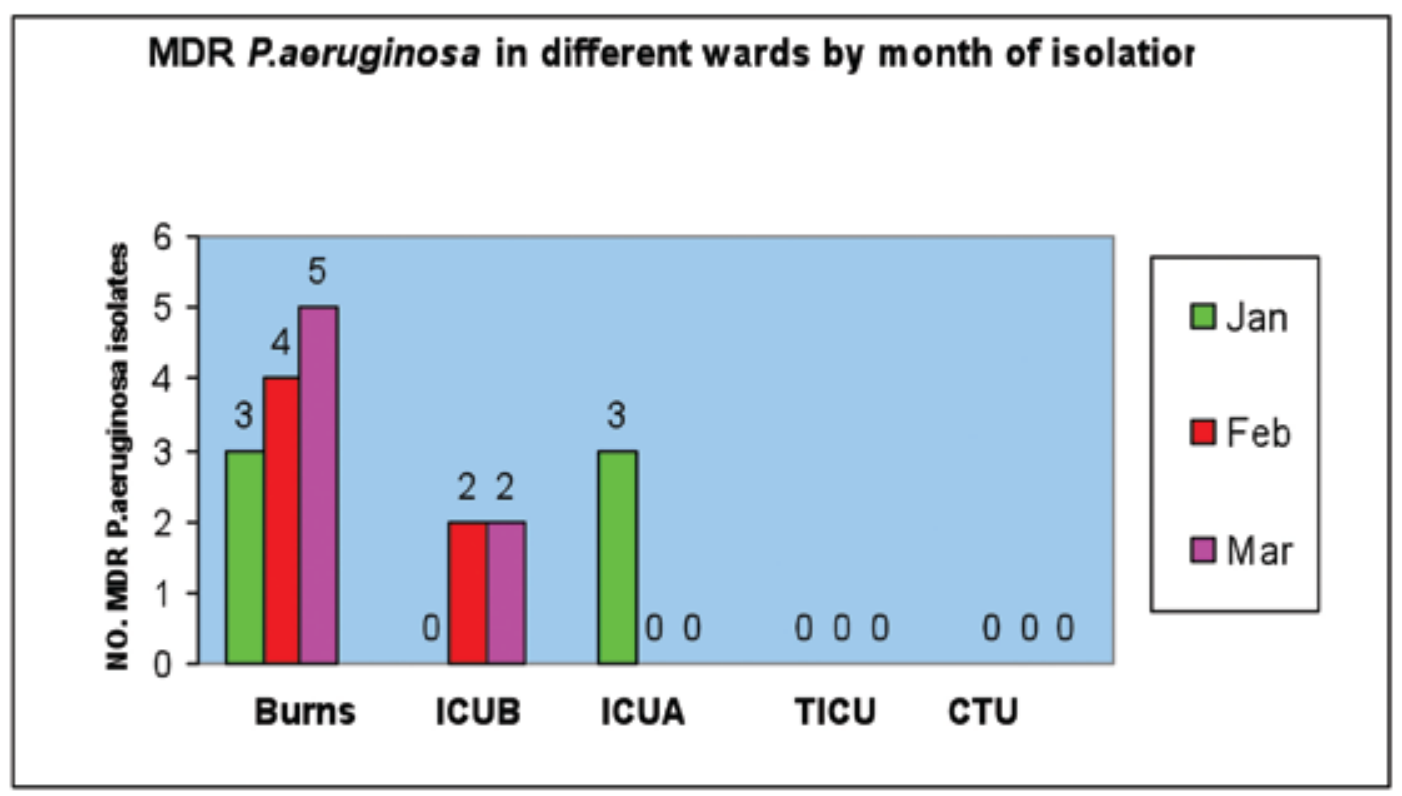

Figure 8. Number of MDR Pseudomonas aeruginosa Histogram 


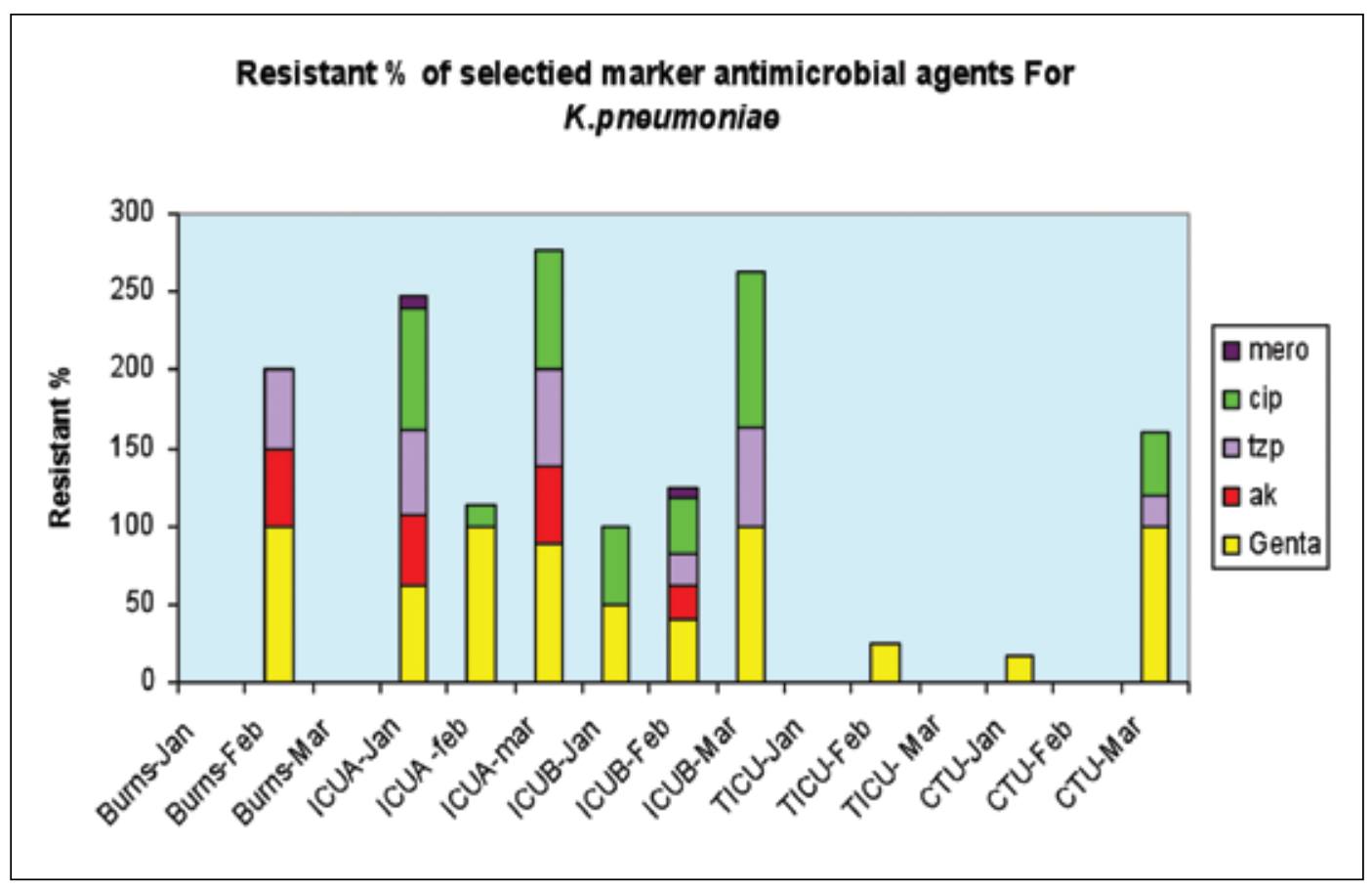

Figure 9. Antibiogram of $K$. pneumoniae from different units within 3 months

The selection of empirical antimicrobial therapy relates to the institution's or unit-specific antibiogram. It had been reported that significant differences exit between the hospital-wide antibiogram and the antibiogram for the individual units. ${ }^{5}$ We also found different antibiogram in each month even at the same ward. Therefore it is important to use direct discussion/ communication with clinician and microbiologist for appropriate antibiotics based on susceptibility result.

We can also suggest continuing to do surveillance of antibiogram and prevalence of MDR organisms not only for infection control but also for empirical therapy based on monthly records.

\section{Burns unit}

We received the different specimens (pus swab, ETA, Blood culture) for MCCS to prepare for grafting and to exclude the infectious causes of sepsis and post graft wound sepsis.

Among the specimens, all targeted bacteria were analysed and we found the prevalence of MRSA (66\%), MDR P. aeruginosa (25\%), were high. These are common potential pathogens in Burns patients. Daily microbiological advice was reported to clinicians, wound management teams and infection control nurses during study period.

The number of MDR Acinetobacter species was 2 each in January, February and only one isolated in March. All were reported as colonization because the patients were stable clinically. We still advised infection control precautions, since Acinetobacter spp has been reported as wound pathogen as well as common potential outbreak organism. ${ }^{6}$

\section{ICUs}

The prevalence of ESBL produced K. pneumoniae was high in ICU A during the study period (table I) and MDR P. aeruginosa (14\%) and ESBL+ K. pneumoniae $(81 \%)$ were significant in ICU B. MRSA was high prevalence $(52 \%)$ in ICU trauma. Because of risk groups of patients in all ICUs, quality management has be included infection control, restriction of antibiotics usage.

\section{Conclusion}

Our findings indicate that the overall prevalence of antimicrobial resistance to the marker antibiotics was high in the bacterial isolates selected in our hospital. The prevalence of resistance varied over the months 
studied and by the type of Unit. Not withstanding the small number of isolates in certain of the ICUs, the very high prevalence of MRSA in the Burns unit and in some of the other ICUs is of major concern. ESBL producing Klebsiella were commonly isolated from most of the ICUs. Emerging resistance to amikacin, ciprofloxacin and even the carbapenems in the ESBL producing Klebsiella is of particular concern. MDR Acinetobacter spp exceeded $50 \%$ over the study period. Although relatively less common, the emergence of multidrug resistant Pseudomonas aeruginosa is a worrying phenomenon.

\section{Recommendations}

Being a major referral hospital for the province of KZN, it is possible that patients were already colonised/ infected at time of admission with antimicrobial resistant bacteria.

We are evaluating baseline screening at first admission, strict hand asepsis, rational use of antimicrobials and continuous education to ward staff. These measures must be reinforced to prevent cross infections and to reduce the mortality rate resulting from nosocomial infections.

It is important to use direct discussion and communication with clinician and microbiologist for appropriate antibiotics based on sense result because of different antibiogram in each month even at the same ward.

We will also continue to do surveillance of antibiogram and prevalence of MDR organisms not only for infection control but also for empirical therapy based on monthly records.

\section{References}

1. Dusé AG. Infection control in developing countries with particular emphasis on South Africa. S Afr journal of epidemiology and infection 2005; 20(2): 37-41.

2. Sein PP, Hoosen AA, Crewe-Brown HH, et al. Antimicrobial susceptibility profile of selected invasive pathogens from academic hospitals in South Africa for the years 2001-2004. S Afr journal of epidemiology and infection 2005; 20: 85-89.

3. Siegel JD, Rhinehart E, Jackson M. Management of multidrugresistant organisms in health care settings. American Journal of Infection Control 2006; 35(10): S165-S193.

4. The Clinical and Laboratory Standards Institute antimicrobial susceptibility testing standards; Sixteenth information supplement. CLSI document M100-S16. Wayne, Pennsylvania, USA, 2008.

5. Binkley S, Fishman NO, LaRosa LA, et al. comparison of unit specific and hospital-wide antibiograms: potential implications for selection of empirical antimicrobial therapy. Inf control and hosp epidemiol 2006; 27: 682-686.

6. Kurcik-Trajkovska B. Acinetobacter spp.- A serious enemy threatening hospitals worldwide. Maced J Med Sci 2009; 2(2): 157-162

\section{Acknowledgments}

Central laboratory (Medical Microbiology Department), NHLS Durban, KZN. 